\title{
Z.U.R. EN EL SUR
}

Carlos ROMAN DEL RIO *

Se equivocó la paloma. Se equivocaba. Por ir al norte, fue al sur.

R. Alberti

\section{OTRA VEZ}

La reciente declaración del área de la Bahía de Cádiz como Zona de Urgente Reindustrialización (Z.U.R.) vuelve a poner sobre el tapete el problema industrial de Andalucía. El Gobierno parece estar decidido a hacer buena su afirmación según la cual el proceso de reconversión de la industria española está en su recta final y en el Boletín Oficial de Estado proliferan las páginas dedicadas al tema. Algunas disposiciones - como esta declaración de Z.U.R. ${ }^{1}$ - afectan directamente a Andalucía, y otras - como las de estímulo al subsector de informática, decididas con posterioridad ${ }^{2}-$ pueden tener $^{2}$ importantes repercusiones sobre su producción industrial. Y todas ellas, unidas a las ya existentes (relativas a SODIAN, IPIA, GAEIA, SOPREA, etc.), vienen a aumentar la frondosidad de la jungla burocrática en la que, con comprensible facilidad, el empresario pierde su camino ${ }^{3}$.

En cualquier caso está claro que son estos los últimos momentos de que disponemos para definir de una manera clara e inequívoca la opción industrial de Andalucía y evitar que una postura pasiva y resignada haga posible la

* Profesor de Economía. Universidad de Sevilla.

1. Real Decreto $189 / 1985$ de 16 de enero (B.O.E. 16 de febrero de 1985).

2. Entre los que se incluyen interés preferente, expropiación forzosa de terrenos, bonificación de hasta el $95 \%$ en el I. T. E. y en la cuota de licencia fiscal, planes especiales de amortización, apoyo especial en los proyectos de I $+\mathrm{D}$, etc.

3. En este sentido hay que destacar el loable intento de clarificación del Instituto de Promoción Industrial de Andalucía que ha ordenado y sistematizado muchas de las referidas medidas de apoyo. Vid. I. P. I. A.: Cuaderno-Guia para la Pequeña y Mediana Empresa. Junta de Andalucía. Sevilla, 1984. 
repetición de pasados desastres. Precisamente alertar sobre ese riesgo -bastante más grave de lo que aparentemente pudiera parecer-es el primer objetivo de las páginas que siguen. Porque si, de una parte no se tienen en cuenta las lecciones de la experiencia y, de otra, se ignora lo que está pasando en un mundo dominado por los países desarrollados en cuya área de influencia se encuentra Andalucía, puede volver a ocurrir en la industria andaluza, a principios de los años ochenta, lo mismo que sucedió a principios de los años sesenta.

En efecto, cuando, después del Plan de Estabilización de 1959, se produjo la incorporación de España al capitalismo internacional, los centros industriales hegemónicos eligieron a Andalucía como blanco de muchas de sus inversiones. Aprovechando la dotación de recursos naturales; la renta de situación; la existencia de mano de obra, sin cualificar pero abundante, barata y poco conflictiva; las ventajas concedidas por la propia Administración Central (que, después de todo, tampoco eran tan diferentes de las que hoy se ofrecen); etc.; la inversión se fue concentrando en empleos muy específicos a partir de los cuales se ha ido conformando una estructura industrial subdesarrollada, dual y dependiente; compuesta por industrias "enclave", desvinculadas de su entorno y vinculadas al exterior; sin tecnología propia; de alto consumo energético; fuertemente contaminantes; poco generadores de empleo y, las más de las veces, limitadas a las primeras fases de los procesos productivos. Las industrias del Polo de Huelva son un excelente ejemplo de cuanto antecede.

¿Es posible evitar que todo eso vuelva a suceder? Sí, si se tiene en cuenta qué es lo que está ocurriendo en las economías industriales avanzadas y se anticipan del modo más preciso posible cuales pueden ser para Andalucía las consecuencias de los cambios que éstas están imprimiendo a sus aparatos productivos. Si se comprenden las claves profundas del funcionamiento de ese mundo y se diseñan un modelo de desarrollo económico propio y una política económica de defensa.

Los países industriales desarrollados han elaborado, y están poniendo en práctica ya, su estrategia para la salida de la crisis por la que atraviesan ${ }^{4}$. En esencia esa estrategia pasa por la actualización y la adaptación a las nuevas circunstancias del modelo de crecimiento industrial que tan excelentes resultados tuvo, para esas economias, en la etapa de expansión 1950-1970. Se trata de seguir caminando, pero aún más intensa y rápidamente que antes, por la senda del desarrollo convencional, introduciendo algunas modificaciones de carácter funcional, que varían según cual sea la opción dominante en cada país de las dos que están en liza - la neokeynesiana y la neomonetarista- en la discusión académica actual. En ambos casos se trata de estimular

4. Vid. Román, C.: "Salida de la crisis por abajo, salida de la crisis por arriba". II Jornadas de Política Económica. Facultad de Ciencias Económicas. Málaga, 1983. 
el crecimiento del producto nacional bruto; luchar contra la inflación y considerar al desempleo como una variable dependiente o "suelta", esperando que el relanzamiento de la producción genere, aunque sea lentamente, nuevos puestos de trabajo. Para conseguir esos objetivos los instrumentos fundamentalmente utilizados son: la contención de los salarios, la "flexibilización" de las plantillas, la introducción de innovaciones tecnológicas, la reducción de los costes energéticos, el uso de una política monetaria rigurosa y/o la reducción de la presión fiscal. El leit motiv es, desde luego, la recuperación de la tasa de beneficios, auténtico estímulo de la inversión privada. Se recomienda confiar en que la reactivación de las inversiones creará, más adelante, empleo. Mientras tanto hay que, resignadamente, "repartir el desempleo".

Hacia finales de 1983 y en el curso de 1984 las medidas señaladas empezaron a surtir sus efectos conteniendose el crecimiento de la inflación y reactivándose la producción en bastantes países, de modo que parece haberse iniciado una cierta recuperación. Existen, sin embargo, razonables dudas sobre la entidad y, sobre todo, la permanencia de esa recuperación. Ninguna duda hay, en cambio, sobre sus costes. En algunos casos, los logros conseguidos en materia de inflación se han pagado en términos de desempleo (el Reino Unido o España podrían ser un buen ejemplo); en otros, el coste de la recuperación se ha repercutido a terceros y más débiles países por la vía del comercio exterior (es el caso de los Estados Unidos con su espectacular déficit de balanza $)^{5}$.

Pero, por lo que a Andalucía respecta, lo más importante de esa estrategia es la redistribución internacional de producciones que implica. Los países desarrollados están reconvirtiendo sus aparatos productivos para concentrar sus esfuerzos en aquellas actividades en las que tienen la posibilidad de ser más eficientes y ganar la delantera en los mercados internacionales. Sus economías se están orientando hacia los llamados "sectores con futuro" 6 , que son precisamente aquellos en los que estos países ostentan un monopolio virtual de la tecnología. Paralelamente se realiza un doble desplazamiento de producciones hacia la periferia. Se trata, por una parte, de aquellas que -por razones de muy diversa índole- ya nos son viables en los países de origen. Son producciones tradicionales, tecnológicamente más atrasadas, de alto consumo energético, con fuerte riesgo ecológico, etc. México, Brasil y algunos países del Sudeste asiático ofrecen buenas muestras de aquellas? ${ }^{7}$. Por

5. Si los Estados Unidos no se deciden a reducir su déficit presupuestario aumentando la presión fiscal y/o conteniendo el gasto público, seguirán demandando ahorro interno y externo para financiarlo, lo que, junto a la política monetaria restrictiva, mantendrá altos los tipos de interés y, con ellos, la cotización del dólar como vehículo del flujo monetario. En estas condiciones el déficit de balanza es difícilmente enjugable.

6. Vid. O. C. D. E.: Interfuturos. Instituto Nacional de Prospectiva. Madrid, 1980.

7. Vid. Balassa, Bela.: The Newly Industrialized Countries in the World Economy. Pergamon Press. New York, 1981. 
otra parte, la estrategia supranacional ha decidido la localización de algunas fases concretas de los nuevos procesos productivos, controlados desde el centro, en determinadas áreas periféricas. Este segundo caso corresponde a las actividades más estrechamente vinculadas a las nuevas tecnologías tales como la electrónica, la informática y las telecomunicaciones en general; la ingeniería genética y la biotecnología; las industrias de la agroalimentación; la tecnología espacial; la tecnología abisal e ingeniería del mar, etc. Los países desarrollados, que controlan el diseño de los nuevos productores y la tecnología de su fabricación, se reservan para sí las etapas finales de los procesos -que es donde más valor se añade- $y$, por supuesto, la comercialización en condiciones de alta competitividad y cuasi-monopolio.

En razón de su situación económica, localización espacial, especialización productiva e, incluso, clima y geografía, Andalucía es excepcionalmente adecuada para la aplicación de las nuevas tecnologías y, otra vez, puede convertirse en receptora de muchas actividades desplazadas desde los países adelantados en unas condiciones que, según y cómo, no harían más que perpetuar indefinidamente su situación de subdesarrollo, marginación y dependencia.

En este sentido, puede decirse que Andalucía está hoy, con respecto a las áreas industriales avanzadas, en una posición muy parecida a la que estaba a principios de los años sesenta. Y la historia puede volver a repetirse si se acepta resignadamente un papel subsidiario en la nueva división internacional del trabajo ${ }^{8}$ impuesto desde aquellas. De igual modo que entonces se decidió localizar aquí buena parte de la industria química básica - con todas sus consecuencias - y otras regiones se reservaron la producción final, ahora puede establecerse una planta de componentes electrónicos, utilizados luego en otro sitio para la fabricación de los ordenadores personales que habrán de ser vendidos más tarde en la Europa comunitaria - de la que Andalucía formará parte- por una compañía multinacional japonesa o norteamericana.

Por añadidura, el espacio económico mundial se concibe hoy de una manera muy amplia y sobre todo, muy flexible, y, dado que el tipo de producciones vinculadas a las nuevas tecnologías es altamente reversible, las instalaciones subsidiarias pueden ser fácilmente desmanteladas si un cambio de las tendencias de la demanda, o de los intereses de la empresa matriz así lo aconseja.

Cualquier intento de reindustrialización o de reconversión que no tenga en cuenta estos hechos contribuirá a que Andalucía vuelva a ser utilizada al servicio del desarrollo de las áreas dominantes, reproduciendo los resultados que hoy se reflejan en la gravedad de su situación económica. Es, pues,

8. Vid. Fröbel, F., Heinrichs, J. \& Kreye, O.: La Nueva División Internacional del Trabajo. Ed. Siglo XXI. Madrid, 1980 . 
inaplazable diseñar una nueva y diferente estrategia de desarrollo industrial que permita sustituir definitivamente al modelo de crecimiento industrial convencional que, por extraño que pueda parecer, sigue aún en plena vigencia. Es absurdo insistir en el error y negarse a admitir las lecciones de la experiencia.

\section{OTRO DESARROLLO}

Por donde hay que empezar es por cambiar la filosofía económica del crecimiento andaluz. Y lo que ese cambio implica es, en esencia, una concepción distinta del desarrollo económico, que lo entienda como un proceso integral no limitado a los aspectos puramente cuantitativos del crecimiento, menos dependiente, más autocentrado y más acorde con las capacidades potenciales endógenas. La nueva estrategia debe concretarse en un Plan, Programa, o como quiera llamarsele, que, siendo mucho más que un paquete de inversiones públicas ${ }^{9}$, establezca de manera inequívoca las orientaciones básicas del desarrollo industrial y especifíque claramente el conjunto de instrumentos y medidas que se pretende utilizar.

Ahora bien, las más elementales reglas de realismo político, económico y político-económico, aconsejan tomar como datos -y no como variables- algunos aspectos fundamentales del problema. Ello significa que continuará correspondiendo al sector privado la función de agente principal de la actividad económica. El sector público puede ayudar, pero eso es todo. Es necesario partir de la idea de que la solución de los problemas económicos regionales no es una responsabilidad que las administraciones central o autonómica puedan, ni deban, asumir en exclusiva. En una economía de mercado tal responsabilidad compete, en primer lugar y por definición, a la iniciativa $\operatorname{privada}^{10}$.

Sin embargo, sí corresponden a ambas administraciones dos importantes atribuciones en relación a la actividad económica privada: la de orientarla y la de promoverla. Y, puesto que, dentro del nuevo marco institucional que consagra el Estado de las Autonomías, los órganos de gobierno regionales han adquirido un notable protagonismo en la definición de la trayectoria que debe seguir el proceso de desarrollo económico en sus respectivos ámbitos territoriales, cumple, en el caso que nos ocupa, a la Junta de Andalucía la misión de diseñar y promover el modelo de desarrollo que los andaluces decidan darse a sí mismos, recogiendo las aspiraciones de los distintos agentes y

9. El Plan Económico para Andalucía es, ante todo, eso. Si a ello se une el hecho de que las exigencias - en buena medida de coyuntura política- de la Reforma Agraria hipotecan sus posibilidades en bastantes terrenos, su impacto sobre la economía andaluza será, en el mejor de los casos, positivo pero insuficiente.

10. Cf. Crouch, C. (Ed.).: State and Economy in Contemporary Capitalism. Croom Helm. London, 1979. Vid. esp. Cap. 6. 
grupos sociales, y asegurando la participación de los responsables directos de la actividad económica ${ }^{11}$.

Para ello, para motivar a la iniciativa privada hace falta, en primer lugar, crear expectativas ciertas de rentabilidad que atraigan a las inversiones y, en segundo, proveer los medios adecuados para que éstas se hagan efectivas. La creación de un clima económico favorable, la dotación infraestructural, los estímulos fiscales y crediticios, etc., son los instrumentos genéricos habituales que la política económica ofrece en estos casos, pero se puede hacer mucho más. En realidad, no hay más límites que los que la realidad imponga a la propia imaginación, ya que entre la simple enumeración de algunas prioridades económicas generales y la elaboración de una cartera de proyectos-tipo concretos existe un amplio espacio intermedio ${ }^{12}$. Hay, pues, que presentar un panorama atractivo, con formulación precisa de los objetivos que se persiguen y de los incentivos que se ofrecen para orientar las inversiones en la forma y modo que más se adapten al tipo de desarrollo elegido. Los empresarios deben saber en qué pueden invertir, cómo y con qué medios cuentan para ello.

\section{OTRO PLAN}

Como es obvio no procede entrar aquí en el esquema jerarquizado de objetivos e instrumentos que habría de constituir el Plan, pero sí resulta pertinente establecer algunas consideraciones generales al respecto. Por las razones expuestas en páginas anteriores, el Plan debería estar situado en el vértice de tres líneas de fuerza decisivas:

1. Las grandes tendencias de las economías desarrolladas dominantes.

2. Los problemas de la economía andaluza.

3 . Sus capacidades reales y potenciales.

11. Cf. Holland, S. (Ed.).: La Superación de la Planificación Capitalista. Oitros-Tan. Barcelona, 1982. Vid. esp. Cap. X.

12. Por cuanto se refiere a instrumentos y medidas como tales, existe una enorme variedad de ellos: Preparación de suelo industrial; canales de comercialización; sistemas de almacenaje; abastecimiento de aguas; electrificación, autopistas, carreteras, caminos vecinales; otras comunicaciones; centros de información y asesoramiento técnico y de gestión empresarial; centros de formación profesional; escuelas; viviendas; centros sanitarios; exenciones, desgravaciones y bonificaciones; ídem. de arbitrios y tasas de las corporaciones locales; flexibilización de los sistemas de amortización; ídem. derechos arancelarios y gravámenes de compensación interior; subvenciones a la inversión; cuotas de la seguridad social; acceso preferente al crédito oficial; ídem. Cajas de Ahorro; ídem. banca privada; otorgamiento de garantías; regulación selectiva de los tipos de interés; ídem. de los coeficientes obligatorios de la banca privada y de las Cajas de Ahorro; flexibilización del pago de los plazos; suministro de inputs en condiciones especiales; institutos de investigación y centros de diseño industrial; agencias para la prestación de servicios colectivos (contabilidad y administración, asesoría fiscal, información sobre precios); apertura de nuevos mercados, ferias, exposiciones... 
En definitiva se trata de conocer y comprender las claves profundas del funcionamiento de la economía mundial ${ }^{13}$, sus estrategias, sus nuevas producciones, sus orientaciones tecnológicas, las tendencias de la demanda en los mercados internacionales, las condiciones de entrada a los mismos, los precios que rigen, etc. para definir el papel que, en ese contexto, Andalucía quiere y puede jugar.

De entre la gran cantidad de consideraciones relevantes en relación a una decisión como ésta destaca lo que nos parece una de las claves fundamentales de todo el problema: la elección de la o las tecnologías apropiadas al tipo de desarrollo elegido.

En este sentido, y en coherencia con las tres líneas de fuerza a las que acabamos de aludir, la nueva estrategia para el desarrollo debe ser planteada a un doble nivel:

\section{A) Estrategia de Alta Tecnología. \\ B) Estrategia de Tecnología Intermedia.}

A) Por fortuna, y como queda dicho, ante Andalucía se abre un amplio campo en el terreno de las nuevas tecnologías y las nuevas producciones. Pero si no empezamos a dibujar ya el futuro perfil de la industria andaluza, éste nos vendrá impuesto. Es urgente prepararse desde ahora para "ganar la delantera" en aquellos de los sectores con futuro en los que Andalucía tiene posibilidades de especializarse y producir eficientemente. Y no basta con definir la estrategia general del nuevo modelo de desarrollo, sino que hay que seleccionar actividades y examinar proyectos concretos. Hay que pensar en otro tipo de productos. Y en otro tipo de empresas ${ }^{14}$. Hay que ir sentando las bases de una nueva estructura productiva.

La gran corporación típica de la etapa del crecimiento industrial convencional, caracterizada por la fabricación, contralizada y rígida, de grandes series uniformes, no es la única forma empresarial ni, probablemente, la más adecuada para las nuevas producciones. Hay que promover un aparato productivo más diversificado, compuesto por muchas medianas $\mathrm{y}$, aún, pequeñas empresas, dispersas pero conectadas entre sí, bien informadas y bien informatizadas, tecnológicamente avanzadas, con flexibilidad para adaptarse al ritmo vertiginoso del cambio en las técnicas de producción, capaces de diseñar sus propios productos diferenciados y de desarrollar procesos productivos completos controlados por ellas mismas, incluida la comercialización. Empresas vinculadas a su entorno, atentas al aprovechamiento de los recursos disponibles, de la cualificación, la experiencia y la tradición, de las fuentes energéticas próximas, de la situación geográfica y, hasta, de la climatología.

13. Cf. Gustafsson, Bo (Ed.).: Post-Industrial Society. Croom Helm. London, 1979, Vid. esp. Cap. 7.

14. Vid. Toffler. Alvin.: La Tercera Ola. Plaza y Jané. Barcelona, 1982. 
También es necesario ampliar nuestro concepto del trabajo, porque cuando éste ha dejado de ser una maldición bíblica para convertirse en un privilegio social, habremos de dar cabida, junto a las tradicionales, a nuevas formas de empleo productivo que incluyan desde el trabajo a domicilio -el nuevo putting-off - hasta los horarios personalizados - el gleit-zeit, $\mathrm{u}$ horario flexible-, pasando por la jornada a tiempo parcial, los empleos interinos y de temporada, los puestos de trabajo compartidos, las sustituciones, el trabajo nocturno, etc. En última instancia, lo importante es hacer el trabajo, no donde o cuando se haga, y estas formas - cuya imagen exagerada sería la de "el ordenador encima de la mesa de camilla"- se adaptan bien a las nuevas producciones que, directa o indirectamente, son las únicas que están creando puestos de trabaho porque, en términos netos, el sector industrial convencional ya no genera más empleo ${ }^{15}$.

En cuanto a los nuevos productos la gama es sorprendentemente extensa, a poco que se aplique la imaginación creativa a la explotación de los recursos regionales. Andalucía está especialmente dotada para la utilización de muchas de las nuevas tecnologías en la agricultura, en la industria y en los servicios. A título puramente enumerativo puede apuntarse a:

- Los cultivos de primor, extratempranos, subtropicales y biológicos; las plantas medicinales y aromáticas, la miel, los cítricos en forma de zumo, los frutos secos, las conservas y la industria agroalimentaria en general.

- La acuicultura, el coral y la minería del mar.

- La vermicultura.

- La producción de algas, abonos orgánicos y fertilizantes, semillas, etc.

- La industria del mármol y del corcho.

- La maquinaria agrícola ligera, sistemas de conservación en frio, etc.

- El textil y la confección de alto diseño.

- Las Artes Gráficas.

- El calzado y las industrias de la piel.

- La artesanía en general.

- La juguetería convencional y electrónica.

- La joyería.

- Los instrumentos de precisión.

- Los ordenadores, computadoras, calculadoras, microprocesadores, procesadores de palabras, semiconductores, sensores, física de los estados sólidos, sistemas ópticos de fibra, etc.

15. En los Estados Unidos, entre 1970 y 1976 , se crearon 9 millones de puestos de trabajo, todos ellos fuera de las 1.000 mayores empresas industriales de la lista de la Revista Fortune, todos ellos en pequeñas y medianas empresas, de las cuales, sólo en 1982, se fundaron 600.000 , frente a las 93.000 de 1950. Naisbitt, John.: Megatrends. Macdonald and Co. London, 1984, pág. 16. 
- La telefonía y televisión por cable. Vídeos.

- Los instrumentos de navegación.

- El reciclaje de residuos sólidos.

- Las energías alternativas, etc.

En el sector de los servicios hay un campo practicamente ilimitado que va desde el turismo y las industrias del ocio hasta el mundo de la investigación y de la información en el sentido más amplio.

B) De otra parte, el uso, con carácter complementario, de la denominada "Tecnología Intermedia" 16 es perfectamente compatible con el tipo de desarrollo que estamos propugnando, y jugaría un papel importante a la hora de reducir el alto grado de dependencia de la economía regional. No se trata de volver a "la autarquía de las aldeas", sino de encontrar soluciones técnicas apropiadas a nuestro espacio y a nuestro tiempo. Con tecnología intermedia no se pueden fabricar productos sofisticados, pero sí es posible satisfacer muchas de las necesidades estrictamente básicas. Carece por completo de sentido que Andalucía siga importando el agua, el pan y la sal.

La evaluación de la aplicabilidad de la tecnología intermedia en la economía andaluza debe arrancar de la consideración de los siguientes presupuestos que son relevantes en relación a los problemas más importantes de la región:

- Aumento del empleo. Los procesos productivos que utilizan tecnología intermedia tienen una gran capacidad para generar puestos de trabajo para los que, además, no es necesaria una alta capacitación, ni de la mano de obra, ni del personal directivo. La escasez de trabajadores con elevada formación profesional y la pretendida falta de

16. La acuñación del término "Tecnología Intermedia" se atribuye a E.F. SCHUMACHER. "Yo la he denominado tecnología intermedia para dar a entender que es muy superior a la tecnología primitiva de épocas pasadas, pero al mismo tiempo mucho más simple, más barata y más libre que la supertecnología de los ricos" (SCHUMACHER, E.F.: "Lo Pequeño es Hermoso". H. Blume Ediciones. Madrid, 1978, pág. 135). Una serie de definiciones posteriores (Vid. MARSDEN, K.: "Progressive Technologies for Developig Countries". Internacional Labor Review. May, 1970 y STEWART, F.: "Intermediate Technology, a Definitional Discussion", en O. C. D. E.: "Choice and Adaptation of Technology in Developing Countries". Development Centre. Paris, 1974) han ido precisando el concepto cuy as características principales son, en resumen, las siguientes:

- Baja relación capital/producto.

- Bajo coste de creación de cada puesto de trabajo.

- Simplicidad del proceso productivo y de su gestión.

- Reducido tamaño de las instalaciones.

- Reducidas exigencias en materia de cualificación de la mano de obra y del personal directivo.

- Utilización de factores productivos locales.

- Bajo consumo energético.

- Bajo consumo de recursos no renovables.

- Escasa contaminación.

- Producción destinada al mercado local o regional.

- Posibilidad de localización en el medio rural.

- Self-help, esto es adecuación a su propio entorno y a sus propios medios con aumento del grado de autonomía y de independencia de cada unidad económica o de cada conjunto de unidades. 
espíritu empresarial en Andalucía podrían ser, en todo caso, limitaciones para el desarrollo de unidades de producción de gran tamaño y funcionamiento complejo que, efectivamente, requieran gran cualificación, pero no para el de las empresas de pequeña y mediana dimensión, basadas en el uso de este otro tipo de tecnología cuyos problemas productivos; de organización; gestión; etc. son comparativamente menores.

- Desconcentración geográfica. Las industrias que trabajan con tecnología intermedia no presentan excesivas exigencias por lo que se refiere a su localización. El abastecimiento y suministro de materia prima pude llevarse a cabo en el entorno próximo; sus menores necesidades infraestructurales; etc. permiten situarlas en el medio rural o semirural, contribuyendo a la descongestión de las zonas urbanas y favoreciendo una ordenación del territorio más racional. De ese modo es posible fijar a la población en sus asentamientos naturales, en tanto que no se trata de que el trabajador se traslade adonde está el puesto de trabajo, sino exactamente al revés.

- Desconcentración sectorial. Este tipo de tecnología hace posible la diversificación de las actividades económicas y la reducción del grado de especialización productiva de las mismas, ampliando la gama de productos y orientándola hacia la elaboración de artículos de consumo básico más generalizado. Ello reduce el nivel de dependencia y aumenta la autosuficiencia de muchos grupos de población que pueden estar perfectamente capacitados para producir una gran cantidad de bienes que habitualmente se ven obligados a importar.

- Articulación del tejido económico. El montaje de una estructura productiva de estas características contribuye a la articulación del tejido económico porque reduce las dificultades -técnicas; financieras; etc.- para el desarrollo de actividades de carácter complementario que, por su mayor interconexión, se sostienen mutuamente y ejercen con facilidad su capacidad de arrastre; lo que es aplicable tanto al propio sector industrial, como a su vinculación con la agricultura y la transformación de los productos de ésta.

- Reducción del grado de extroversión. La diversificación de la producción y la disminución de la dependencia del exterior en cuestión de bienes de equipo; de materia prima; de mano de obra; etc., así como la orientación de la producción final hacia el mercado local o regional, reducen el grado de extroversión y promueven un desarrollo económico más autónomo y autocentrado, lo que, de algún modo, resta importancia a la posible menor competitividad en otros mercados de los productos elaborados en esas condiciones. 
- Defensa del medio ambiente. La tecnología intermedia utiliza fuentes de energía renovable; aprovecha los recursos naturales de su entorno; es "ecológicamente respetuosa" y permite el reciclaje de los desechos en mucho mayor medida que la tecnología altamente sofisticada. Los costes sociales de reparación no existen porque la agresión al medio ambiente es mínima.

La objección habitual al uso de tecnología intermedia suele centrarse en el tema de la competitividad ${ }^{17}$. Pero las afirmaciones al respecto deben ser cuidadosamente matizadas, porque es muy importante tanto la clase de productos a los que se aplica - sirve para unos y no para otros-como el mercado -local o comarcal- de los mismos. Además, el aumento de la competitividad no sólo se consigue por la vía de la producción en serie, sino que también es posible ser eficientes reduciendo los costes de capital, energéticos, de transporte, de comercialización y publicidad, mejorando la calidad ${ }^{18}$, disminuyendo el despilfarro, etc. Y, lo que no es menos importante, la productividad de la fuerza de trabajo también es mayor si lo es su participación en el proceso de toma de decisiones. Y, en última instancia, aunque ronde el fantasma del proteccionismo, cabe el recurso a la subvención. Si nadie se escandaliza cuando se subvenciona el desempleo, que nadie lo haga cuando se subvencione el empleo.

Hay, pues, alternativas, pero habrá que encontrar la combinación más adecuada porque no existe una opción única y definitiva. Lejos de las fórmulas utópicas, rotundas y cerradas, hay que buscar soluciones posibles, múltiples y flexibles, que se hagan y se deshagan en una continua adaptación a la realidad cambiante.

Las dos estrategias sugeridas son muy distintas, pero no mutuamente excluyentes, y en ambas el sector público tiene mucho que hacer. Al él corresponde definir cuidadosamente los respectivos ámbitos de aplicación y hacerlas compatibles; elaborar los planes de actuación y controlar su ejecución. Pero para eso tiene que dejar de ser un freno burocrático y convertirse en un promotor de iniciativas. Suya es la responsabilidad de que la imaginación sustituya a la rutina.

17. Vid. Emmanuel, Arguiri.: Appropiate or Underdeveloped Technology? John Wiley \& Sons. Chichester, 1982.

18. Vid. Harris, Marvin.: La Cultura Norteamericana Contemporánea. Alianza. Madrid, 1984. 